\title{
The relationship between social identity and cognitive diversity in environmental stakeholders
}

Payam Aminpour*1,2, Heike Schwermer ${ }^{3,4}$, and Steven Gray ${ }^{2,5}$

${ }^{1}$ Department of Environmental Health and Engineering, Johns Hopkins University, Baltimore, MD 21218, USA.

${ }^{2}$ Collective Intelligence Research Group, IT University of Copenhagen, København, Denmark. ${ }^{3}$ Institute of Marine Ecosystem and Fishery Science, Center for Earth System Research and Sustainability, University of Hamburg, Hamburg, Germany.

${ }^{4}$ Department of Economics, Center for Ocean and Society, Christian-Albrechts-University Kiel, Germany.

${ }^{5}$ Department of Community Sustainability, Michigan State University, East Lansing, MI 48824, USA.

* Corresponding Author: payamaminpor@gmail.com 


\section{Abstract}

Groups with higher cognitive diversity, i.e. variations in how people think and solve problems, are thought to demonstrate improved performance in complex problem-solving. However, embracing or even engineering adequate cognitive diversity is not straightforward and may even jeopardize social inclusion. In fact, those that want to promote cognitive diversity might make a simplified assumption that there exists a link between identity diversity, i.e. range of social characteristics, and variations in how people perceive and solve problems. If it holds true, incorporating diverse identities may concurrently satisfy desirable cognitive diversity while social inclusion is explicitly acknowledged. However, currently there is a lack of empirical evidence to support this hypothesis in the context of complex social-ecological systems-a system wherein human and environmental dimensions are interdependent, where common-pool resources are used or managed by multiple types of stakeholders. Using a fisheries example, we examine the relationship between resource stakeholders' identities and their cognitive diversity. We used cognitive mapping techniques in conjunction with network analysis to measure cognitive distances within and between stakeholders of various social types (i.e. identities). Our results empirically show that groups with higher identity diversity also demonstrate more cognitive diversity, evidenced by disparate characteristics of their cognitive maps representing their understanding of the fishery dynamics. These findings have important implications for sustainable management of common-pool resources, where the inclusion of diverse stakeholders is routine, while our study shows it may also achieve higher cognitive coverage that can potentially lead to more complete, accurate, and innovative understanding of complex resource dynamics.

Keywords: Cognitive diversity, identity diversity, collective intelligence, social-ecological systems, cognitive mapping, complex network analysis 


\section{Introduction}

Diversity is a term generally used to identify differences between individuals or describe instances of being composed of differing elements or including different qualities. Depending on the type of differences by which the diversity is determined, people can be categorized under demographic, cultural, political, occupational, intellectual, or many other categories. As a guiding principle, these dimensions can be dichotomized into two overarching kinds of diversity: (a) identity diversity and (b) cognitive diversity (1-3).

Identity diversity—also known as surface-level diversity_refers to differences in a set of subjective characteristics that are apparent across individuals or groups (2). As such, many social categories or deductive specifications that are explicitly defined by demographic, socioeconomic, cultural, political, or any other salient features of the individuals fall into identity diversity. These are factors that are generally considered observable (think demographic categories), and often perceptible by those who seek or care about diversity and inclusion (4).

On the other hand, cognitive diversity-also known as deep-level diversity—refers to differences in how people represent, think about, and solve problems (2). Hong and Page (2004) refer to this kind of diversity as functional differences and explain how it might be determined by measuring variations in people's perspectives (i.e., how they represent a problem) and heuristics (i.e. how they find solutions to a problem) (also see (5)). This kind of diversity has been suggested to be a critical driver of improving group performance in complex problemsolving (6-8). Three mostly cited problem-solving benefits associated with cognitive diversity are i) augmentation (i.e., the generation of a larger pool of knowledge), ii) purification (i.e., the cancelation and refinement of errors and inaccuracies mostly in predictions), and iii) recombination (i.e., the emergence of innovative solutions as a result of higher possibility for permutation and combination of knowledge) (6,9-11). Accordingly, since human societies face 
more complex problems today, cognitive diversity becomes a vital ingredient in contemporary problem-solving.

Despite these benefits, achieving cognitive diversity is not always straightforward because differences across groups and individuals are not immediately observable or readily detectable (12). Instead, to assess cognitive diversity, researchers need to dive deeper into invisible variations in personality, intellectual abilities, and cognitive characteristics of individuals using intelligence tests (13), psychological and neuropsychological assessments (14), mental modeling techniques (15), or cognitive ability tests (16). Yet, seeking and embracing cognitive diversity does not necessarily satisfy the full inclusion of diverse social identities $(2,17)$, which can be problematic wherein social inclusion is vital to achieving ethical goals such as achieving social equity and resolving conflicts in areas like participatory governance (18).

However, the inverse may possibly be true —-that is, some sort of identity diversity can congruently achieve beneficial cognitive diversity $(19,20)$. Under certain circumstances, incorporating diverse identities into problem-solving may concurrently encourage cognitive diversity which is beneficial to groups' problem-solving capability, while it also satisfies the social equity goals. While it does not appear to be an unreasonable assumption in some cases (e.g., particularly those cases wherein some salient differences that determine identity diversity are of high problem-solving relevance) (21), the literature around "diversity" is still open to debate about the relationship between identity and cognitive diversity. In fact there is evidence to the contrary, that is, that identity diversity does not always contribute to beneficial cognitive diversity $(2,3,17)$.

Regardless of these controversies, in many cases, achieving both kinds of diversity at the same time or what has been referred to as "congruence between surface and deep-level characteristics" - has been thought to be a major success (2). Despite practical challenges, implementing this congruency has been recommended in multi-stakeholders governance and 
management systems such as common-pool resources and environmental assessments (18). Understanding diversity within stakeholders who interact with natural resource systems, therefore, serves as as a great case study to evaluate these congruencies since they typically involve multiple stakeholders and require the participation of socially diverse groups in dialogue, deliberation, decision-making, and adaptive co-management (22). This inclusive participation of socially diverse groups of stakeholders in decision-making and policy development processes instills stakeholders' sense of ownership of the decisions, helps them address conflicts and build shared understandings, thereby improving the the legitimacy of natural resource management strategies $(23,24)$. It thus constitutes an important component of improving decision-making and social and environmental sustainability (25).

In addition, natural resource systems are composed of both social components (i.e., humanrelated factors like consumption, regulations and conservation) and ecological components (i.e., nature-related factors like ecosystem health, resource abundance, productivity), as well as their feedback interactions (e.g., the impact of consumptions or regulations on resource dynamics or the impact of degraded ecosystem productivity on human well-being). These so-called socialecological interdependences commonly lead to complex system behaviors and dynamics that are hard to predict $(26,27)$. As a result, understanding and managing natural resource systems typically entails the participation of cognitively diverse individuals who bring a wider range of perspectives and heuristics to the table, and their diverse knowledge pool can lead to a greater cognitive coverage and a boosted problem-solving capability $(7,8,28,29)$.

Here we explore whether congruence exist in groups with diverse stakeholders who self-identify themselves in different professional roles by which certain type of interactions with, or use of, natural resources are specified. We hypothesized that different social identities are associated with certain cognitive spaces and knowledge (i.e. there exist a correlation between surface and deep-level diversities). 
We build this hypothesis on prior theoretical and empirical evidence describing that different social groups of resource stakeholders (e.g. fishermen, hunters, scientists, policymakers, and managers) interact differently with natural and social dimensions of ecosystems at different time and spatial scales. Such different social groups may also be subjected to diverging beliefs and values (30), disparate experiences with the nature (28), differences in preferred adaptation strategies and management policies (31), and are thought to build in their minds diverse cognitive representations (i.e., mental models) of the system that reflect their specific interests and interactions.

To empirically support our hypothesis, we use a fisheries example where multiple groups of stakeholders interact differently with a natural ecosystem (i.e., a common-pool resource system). Our case is the Western Baltic cod (Gadus morhua) in Germany. Western Baltic cod is of crucial importance for regional ecosystems and constitute a vital component of coastal economies $(32,33)$ (a more detailed explanation of the case study is provided in Supporting Information). Cod is known as one of the species in high demand and plays a key role in the Baltic Sea, environmentally, socially and economically (34). Here, we focus on stakeholder groups who are differently affected by or involved in fisheries management and therefore represent varying interdependences with the natural ecosystem (Supporting Information, Fig. S1).

We use a semi-quantitative cognitive mapping technique called Fuzzy Cognitive Mapping (FCM) $(35,36)$ in conjunction with network analysis to develop a novel approach to measuring cognitive distances within and between social groups of stakeholders (i.e. individuals with diverse roles and resource use). Finally, analyzing the congruence of differences in stakeholders' identities and features of their cognitive maps can empirically demonstrate the potential proximity of surface and deep-level diversities among environmental stakeholders. 


\section{Methods}

\section{Data collection}

\section{Mental models and fuzzy cognitive maps}

To measure variation in stakeholders' perceptions and understanding of the complex socialecological relationships (i.e. deep-level diversity), we collected individual mental models about fisheries ecosystem dynamics and management from fisheries stakeholders. Theoreticians have hypothesized that humans develop in their mind simplified internal representations of the complex reality that allow them to perceive the world around them (15). Individuals who observe, interact with, and experience the world around them can concurrently develop an internal model of the external world to understand it and predict how it functions (37). These socalled mental models represent patterns of perceived cause-and-effect relationships among various concepts that are built through reasoning and thus shape the basis for problem-solving and decision-making (38). Importantly, these mental models can be elicited through cognitive mapping techniques (39). Cognitive maps are graphical representations of mental models in the form of directed networks where nodes represent concepts and edges show the causal relationships between them (Supporting Information, Fig. S2)

Here we used Fuzzy Cognitive Mapping (FCM) (35)—an enhanced form of cognitive maps which mathematically and graphically model system components (nodes), their causal relationships (edges), and the strength of these relationships using a normalized quantitative parameterization of causal magnitudes. In an FCM, edges are characterized by a normalized number in the interval of $[-1,+1]$, corresponding to the strength and sign of causal relationships between nodes, thereby forming a weighted directed graph (40). These weighted directed graphs can be analyzed using complex network analysis through measures and algorithms 
related to node connectivity, graph distances, their adjacency matrices similarity, and graph clustering (41).

\section{Cognitive map elicitation}

Five relevant stakeholder types were identified in a stakeholder analysis: Local fisheries (including commercial and recreational fishers), representatives of tourism industry, Nongovernmental organizations (NGOs), managers and policymakers, and scientific experts. Two key criteria were applied to sample study participants $(N=33)$ using a purposeful sampling strategy: stakeholders needed to be affiliated with a German institution either through their job or honorary position, and have been active (involved or affected) in the cod fishery in the Western Baltic Sea for more than 5 years (see the description of interviewed stakeholders in Supporting Information, Table S1). We elicited stakeholders' FCMs through semi-structured interview processes. This study was conducted with approval of University of Hamburg's Institutional Review Board, and informed consent was acquired from all participants. Individuals were asked to identify relevant concepts (i.e., system components) and their causal relationships, from which they then drew a concept map representing their mental models about Western Baltic cod ecosystem and fisheries management. This process included routine FCM data collection practices with open-ended concepts (42). Participants' cognitive maps were digitized after the interview (i.e. maps were converted to digital weighted directed graphs using www.mentalmodeler.org) and sent back to the interviewees for validation. We described in details the cognitive map elicitation process elsewhere (43) and in Supporting Information (Fig. S2).

\section{Data analysis}

\section{Comparing graphs}


To measure cognitive diversity, we perform network comparisons by defining a measure of distance between $\mathrm{FCM}$ graphs. Each FCM is a directed, weighted graph $G(V, E)$, with $V$ being the set of nodes (i.e. concepts) and $E$ being the set of edges (i.e., causal connections). We compute the distance between a pair of FCMs by taking into account two measures:

a) The distance between the adjacency matrices of their graphs:

The adjacency matrix $A$ of a graph $G$ is a $n \times n$ square matrix, where $n$ is the number of nodes, and the elements of the matrix $\left[a_{i j}\right]$ indicate whether pairs of nodes $i$ and $j$ are adjacent $\left[a_{i j}\right]=$ 1 or not $\left[a_{i j}\right]=0$ in the graph. One common norm used as graph distance is the Jaccard distance (44). Given two graphs $G_{1}\left(V_{1}, E_{1}\right)$ and $G_{2}\left(V_{2}, E_{2}\right)$ with adjacency matrices $A_{1}$ and $A_{2}$, the Jaccard coefficient $J$ is defined as $J\left(A_{1}, A_{2}\right)=\frac{A_{1} \cap A_{2}}{A_{1} \cup A_{2}}$, and their Jaccard distance is calculated as follows:

$$
d_{J}=1-J\left(A_{1}, A_{2}\right)
$$

b) The distance between the spectra of their graphs:

The spectrum of a graph $G(V, E)$ is the set of eigenvalues of its normalized Laplacian $(45,46)$ and contains useful information about the principal properties and structure of a graph which has important implications for graph comparisons (46-48). Let $A_{w}$ be the undirected, weighted adjacency matrix such that the elements of $A_{w}=\left[a_{i j}\right]=\left[a_{j i}\right]$ indicate the edge weights between pairs of nodes $i$ and $j$ that are adjacent in the graph. Then the (symmetric) normalized Laplacian is defined as $L^{\text {sym }}=D^{-1 / 2} L D^{-1 / 2}$, where $L=D-A_{w}$, while $D$ is the degree matrix. Importantly, all eigenvalues of the normalized Laplacian are real and non-negative (46), thereby offering a practical tool for measuring graph distances. Given two graphs $G_{1}\left(V_{1}, E_{1}\right)$ and $G_{2}\left(V_{2}, E_{2}\right)$ we find a set of all eigenvalues for each normalized Laplacian as their spectra. Similar to the approach outlined in $(49,50)$, we compute the Euclidian distance between the graphs' spectra $d_{s}$ as follows: 


$$
d_{s}=\sum_{i=1}^{k^{*}}\left(\lambda_{1 i}-\lambda_{2 i}\right)^{2}
$$

where $\lambda_{i}$ is the $i^{\text {th }}$ largest eigenvalue and $\left(\lambda_{i} \geq 0\right.$ for $\left.\forall i\right)$. We find the smallest $k$ such that the sum of the $k$ largest eigenvalues constitutes at least $90 \%$ of the sum of all of the eigenvalues. If the values of $k$ are different between the two graphs, we use the smaller one $k^{*}$.

These two measures of graph distance are complementary as they take into account the structural properties that are characterized by either edge directionality or edge weights. Thus, to jointly acknowledge the weight and directionality of causal connections in FCMs, we define the cognitive distance between two FCMs as follows:

$$
C D=\frac{d_{s}}{1-d_{J}} \times \varphi
$$

were $\varphi$ is the standardization coefficient for mapping $C D$ to a normalized range between $[0,1]$.

All individual cognitive maps were converted into adjacency matrices and the cognitive distances between any pairs of maps were computed using equation 3. For each identifiable social group (e.g., fishers, managers, NGOs, tourism, and experts) we make two sets of cognitive distances: the intra-group set including the cognitive distances between any pairs of socially homogeneous individuals who share the same social category (e.g., a pair of fishers), and the inter-group set including the cognitive distances between any pairs of socially diverse individuals who do not share the same social category (e.g., a pair of one fisher and one manager). Independent sample t-tests were used to compare the means of cognitive distances in intra-group and inter-group sets. This helped us determine whether or not inter-group distances were longer than intra-group distances—-that is, the cognitive diversity amongst socially diverse individuals was statistically significantly higher than the cognitive diversity in socially homogeneous ones. 


\section{Monte-Carlo method}

We then used the Monte-Carlo method (MCM), wherein the virtual FCMs were randomly reproduced from the probability distributions of stakeholder-driven cognitive maps. That is, virtual agents with a defined identity (e.g., fisher, manager, etc.) were computationally generated, such that their cognitive maps were randomly drawn from the probability distribution of FCMs elicited from actual individuals of that social type (8). Importantly, the MCM helps us regenerate virtual samples of stakeholders that artificially represent various levels of identity diversity, thereby enabling us to carry out a probabilistic examination of how identity diversity correlates with cognitive diversity. Using MCM we built 100 replicates of our FCM sample. Each reproduced sample has $N=33$ individuals (to resemble actual sample size) with a random combination of virtual agents from different social categories (i.e. individuals of different types). For each random replicate, 1000 bootstrap resamples were used to estimate the $95 \%$ confidence interval.

To measure the identity diversity of each reproduced sample we used Shannon's entropy index $(H)(51)$. The Shannon's entropy index takes into account both the richness (i.e., how many unique identities exist in a sample) and the evenness (i.e., how even the proportions of stakeholder identities are in a sample), and thus provides useful information about identity diversity. Fig. 1 displays four illustrative samples of size 10 with different richness and evenness. We calculate identity diversity in each sample using the following equations:

$$
\begin{gathered}
H=-\sum_{i} p_{i} \times \operatorname{Ln}\left(p_{i}\right), \quad p_{i}=\frac{n_{i}}{N} \\
D=\frac{e^{H}}{\max (r)}
\end{gathered}
$$


where $H$ is the Shannon's entropy index, $n_{i}$ is the number of individuals of type $i, N$ is the sample size, $D$ is the identity diversity, and $\max (r)$ is the maximum possible richness (i.e. maximum possible number of unique types) in a sample, which is 5 in our case. $D$ is a number between $[0,1]$ with values closer to one representing higher diversity. In addition, we define cognitive diversity as the mean of pairwise cognitive distances $(C D)$ (see equation 3) between any two individuals within the sample. Finally, the correlation between identity diversity and cognitive diversity were calculated using Pearson correlation coefficient.

A

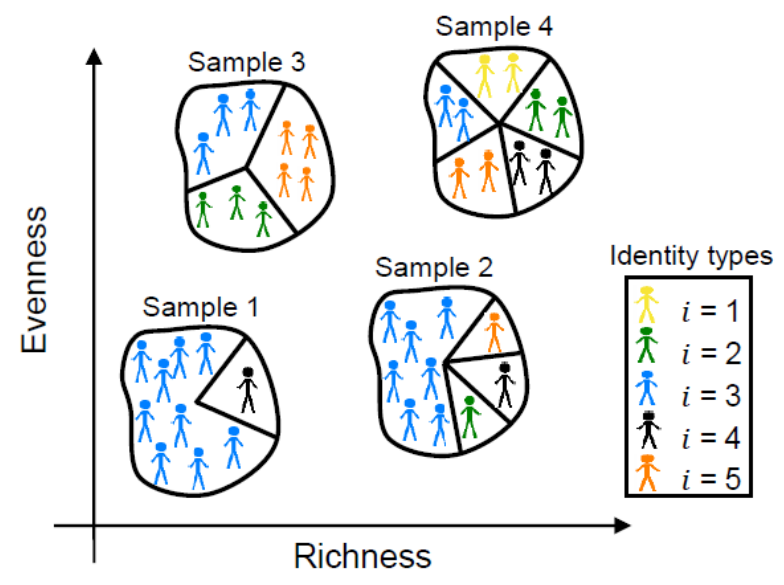

B

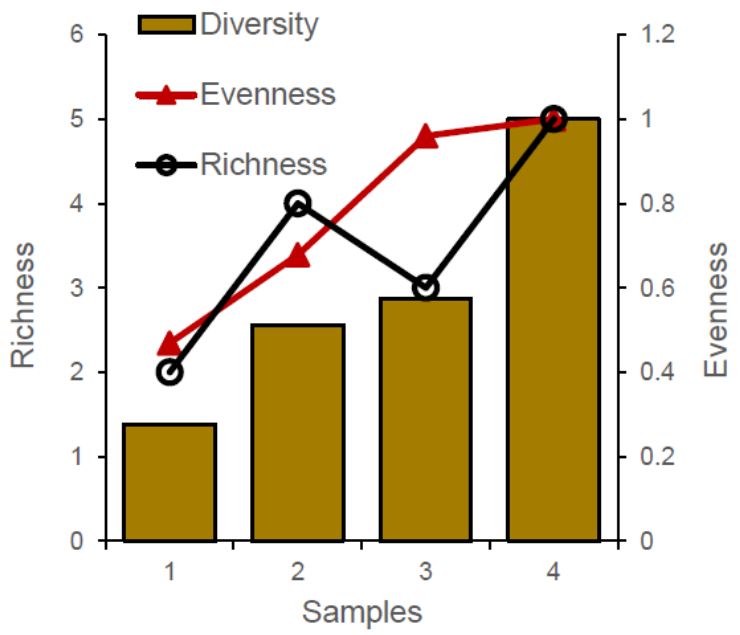

Fig 1. Illustrative samples of individuals with different levels of richness and evenness. Four hypothetical examples with low-to-high levels of richness and evenness are shown in (A). The calculated identity diversity with regards to each sample's richness, evenness, and their influence on the level of diversity is shown in (B). Samples' diversity were calculated using an information theoretic measure built on Shannon's entropy formula.

Last but not least, we drew on network theory and cognitive map analyses of perceived causation (52) to cluster FCMs using their network micro-motifs (i.e., micro-structures that are constructed by two or three nodes and some unique patterns of connections between them, which shape the underlying elements of perceived causation in a cognitive map). The frequency distribution of these micro motifs in one cognitive map -also known as directed graphlets of 
size two and three-can provide useful information about how one individual sees the causal interdependencies and can be used as a tool for deep-level comparisons (Tantardini et al., 2019). Theoretical and empirical studies have frequently suggested the use of 7 simple micromotifs (see Table 1) to exemplify common patterns of perceived causation (52-58). We combined Principle Component Analysis (PCA) and K-mean clustering to develop an unsupervised learning algorithm that clusters individuals based on their frequency distributions of these 7 micro-motifs and no pre-defined labeling. We also clustered the individuals based on their pre-defined identities (i.e. social types labeling). Analyzing and visualizing the alignment between identity-based clustering and graphlet-based clustering helped us further examine the proximity of surface and deep level diversities.

Table 1. Seven micro-motifs exemplifying common patterns of perceived causation in cognitive maps.

\begin{tabular}{|c|c|c|c|}
\hline $\begin{array}{l}\text { Typology of } \\
\text { causality }\end{array}$ & $\begin{array}{l}\text { Corresponding network } \\
\text { structure (Graph/et) }\end{array}$ & $\begin{array}{l}\text { Micro- } \\
\text { motifs }\end{array}$ & Details \\
\hline $\begin{array}{c}\text { Direct } \\
\text { dependence }\end{array}$ & Simple edge & & One node affects an adjacent node \\
\hline Bi-directionality & Closed pair & & $\begin{array}{l}\text { One node affects and is affected by an adjacent } \\
\text { node }\end{array}$ \\
\hline Multiple effects & Linear triplet (Source) & & $\begin{array}{l}\text { Two non-adjacent nodes are affected by a shared } \\
\text { adjacent node }\end{array}$ \\
\hline Indirect effect & Linear triplet (Passer/Flipper) & & $\begin{array}{l}\text { One node affects a non-adjacent node throw a third } \\
\text { node that mediates the effect }\end{array}$ \\
\hline Multiple causes & Linear triplet (Sink) & & $\begin{array}{l}\text { Two non-adjacent nodes affect a shared adjacent } \\
\text { node }\end{array}$ \\
\hline Moderated effect & Closed Triplet (Feedforward) & & $\begin{array}{l}\text { One node affects an adjacent node while it } \\
\text { simultaneously affects that node throw a third node. }\end{array}$ \\
\hline Feedback loop & Closed Triplet (Feedback) & & $\begin{array}{l}\text { Three adjacent nodes affect one another through a } \\
\text { cycle, either clockwise or counter-clockwise }\end{array}$ \\
\hline
\end{tabular}




\section{Results}

\section{Intra versus inter-group cognitive distances}

We collected 33 FCMs through semi-structured interviews with stakeholders (see supplementary information). Five social groups (i.e. types) of stakeholders participated in our study. Fig. 2 illustrates the comparison of intra-group versus inter-group pairwise cognitive distances (see equation 3). Independent sample t-tests were used to compare the means, and

p-values demonstrate significance of their difference. In all five socially distinguishable groups of stakeholders (Fig. 2 A-E) the mean of inter-group cognitive distances is longer than the mean of intra-group cognitive distances, and in three groups (i.e., NGOs, tourism, and experts) these differences are statistically significant at the level of $p<0.05$. It is visible from the Fig. $2(\mathrm{~F})$ that, once all individuals are combined, the cognitive diversity (measured by the mean of cognitive distance between any pairs of individuals) amongst socially diverse individuals (i.e. inter-group pairs) is statistically significantly higher than the cognitive diversity in socially homogeneous ones (i.e., intra-group pairs).

\section{Correlation of identity and cognitive diversity}

Next, we examined the correlation of identity and cognitive diversity using the MCM. Fig. 3 shows the result of 100 randomly generated samples of stochastic agents (i.e. artificial individuals who own randomly-generated cognitive maps drawn from the probability distribution of actual FCMs). These random samples represent different levels of identity diversity determined by equation 5 . Pearson correlation coefficient of 0.74 revealed a positive association between samples' identity diversity and the mean of pairwise cognitive distances among agents' cognitive maps. That is, samples high in identity diversity are $74 \%$ probable to show high 
cognitive diversity (each sample was bootstrapped 1,000 times to estimate $95 \%$ confidence interval).
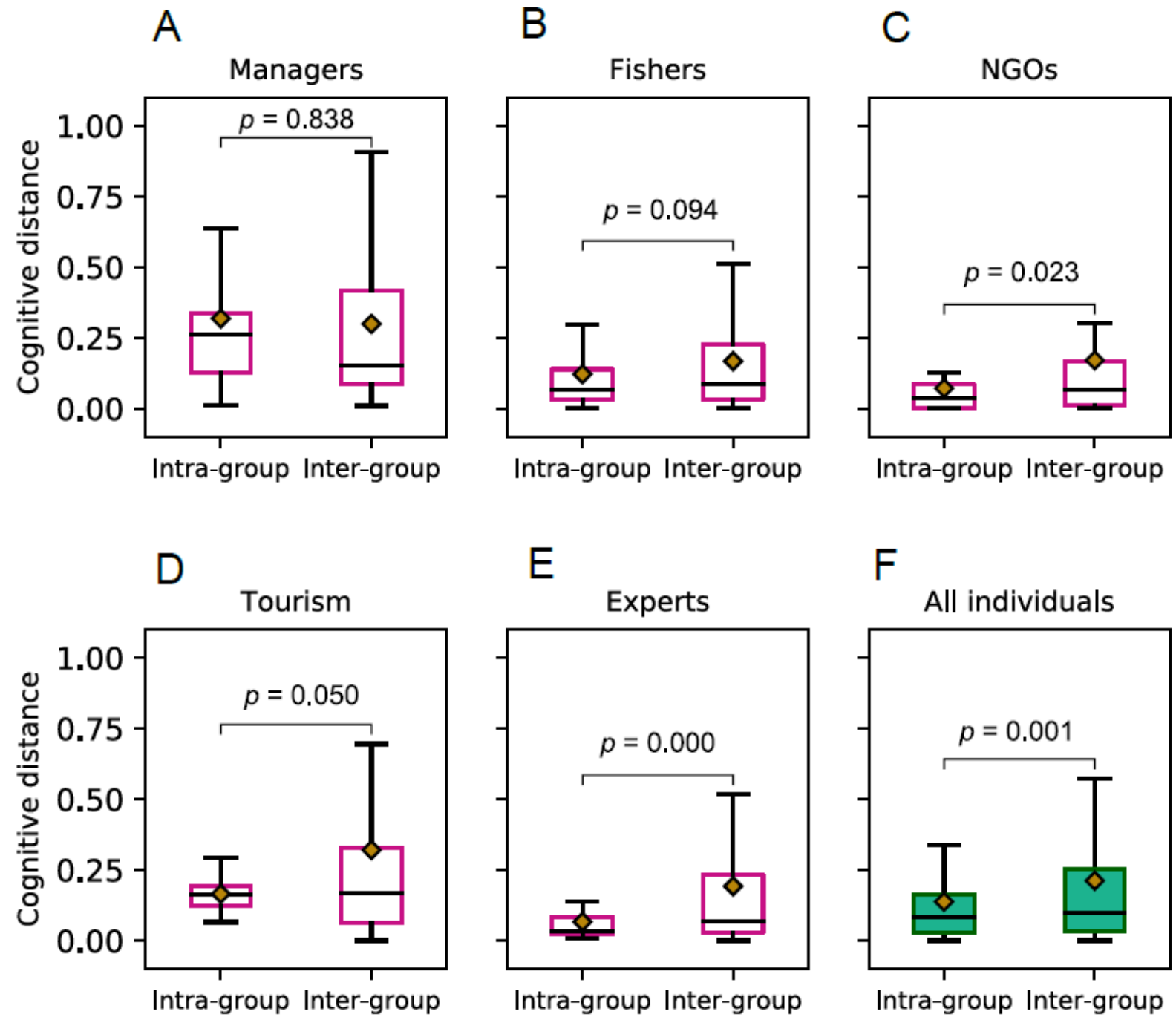

Fig 2. Comparison of intra-group (i.e., within social groups of stakeholders) versus intergroup (i.e., between social groups of stakeholders) pairwise cognitive distances.

Independent sample t-tests were used to compare the means, and $p$-values demonstrate significance of their difference. (A-E) show the mean of inter-group versus intra-group cognitive distances for five social groups of stakeholders. Overall results for all individuals is shown in $(F)$. 


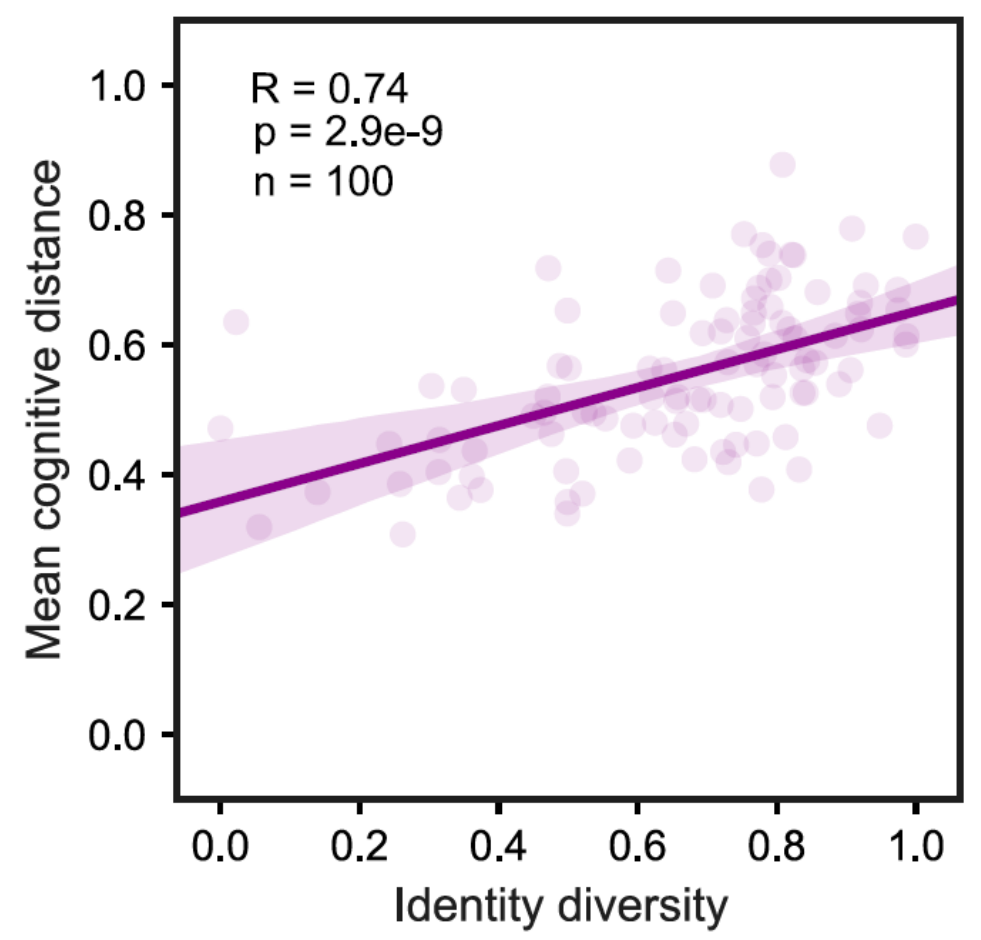

Fig 3. Correlation between identity and cognitive diversity. The figure shows the result of 100 randomly generated samples of size $\mathrm{N}=33$ individuals. The shaded area represents $95 \%$ confidence interval estimated by bootstrapping the sample 1000 times.

\section{Proximity of surface and deep-level clusters}

In addition, we compared the results of two clustering algorisms: one based on predefined socially distinguishable labels (i.e. identity), and the other one based on an unsupervised dimension reduction technique (i.e., a PCA) that transforms cognitive maps from their 7dimensional micro-motif space (see Table 1) to a 2-dimensional principle component space, where clusters are determined by k-nearest neighbors (based on their Euclidian distance). Figs. $4 \mathrm{~A}$ and $\mathrm{B}$ illustrate the results of these two clustering algorithms for a randomly reproduced sample of size 1,000 (with 200 individuals for each of five social groups). It is visible from these figures that individuals who are similarly clustered by their predefined social identities are more likely to be in the same cognitive neighborhood that represents a prevailing cluster of individuals who are closely matching in terms of how they perceive causal interdependences. Fig. $4 \mathrm{C}$ 
shows the probability of possible concurrencies formed by the categories of two clustering algorithms. Interestingly, for each cognitive cluster, there exists one and only one dominating social cluster (i.e. identity) whose concurrency probability is greater than 0.5 , meaning that the overwhelming majority of individuals within a cognitive cluster share the same social identity. These findings revealed that environmental stakeholders demonstrate distinguishable differences in discrete aspects of their cognitive models (i.e., deep-level clusters) that are most probably aligned with the way they could have been distinguished by their disparate social identities (i.e., surface-level clusters). Consequently, these perfect alignments demonstrate the strong likelihood of congruence of surface and deep level diversities in environmental stakeholders.

\section{Discussion}

The importance of diversity, in general can be seen across systems, from ecosystems (59) to economic systems (60), and also extend to norms regarding social inclusion and social equity $(61,62)$. In each case, diversity is considered to make systems more adaptable and resilient to changes. Here we extend this general notion of the diversity bonus (4) and provide evidence of the hypothesized correlation between identity diversity (surface) and cognitive diversity (deep). Our data provide empirical evidence that the inclusion of diverse stakeholder groups in natural resource problem-solving has implications for better understanding the complexity of natural resource systems since different social groups interact within these systems somewhat similarly by group, but distinct across groups providing more opportunities for full cognitive coverage (8). While the literature on collaborative natural resource management has for some time promoted the inclusion of diverse stakeholder groups and public participation for improved decisionmaking (18), the diversity of knowledge systems that these different social groups bring with them has been largely assumed, rather than empirically evaluated with some exceptions (63). 
A

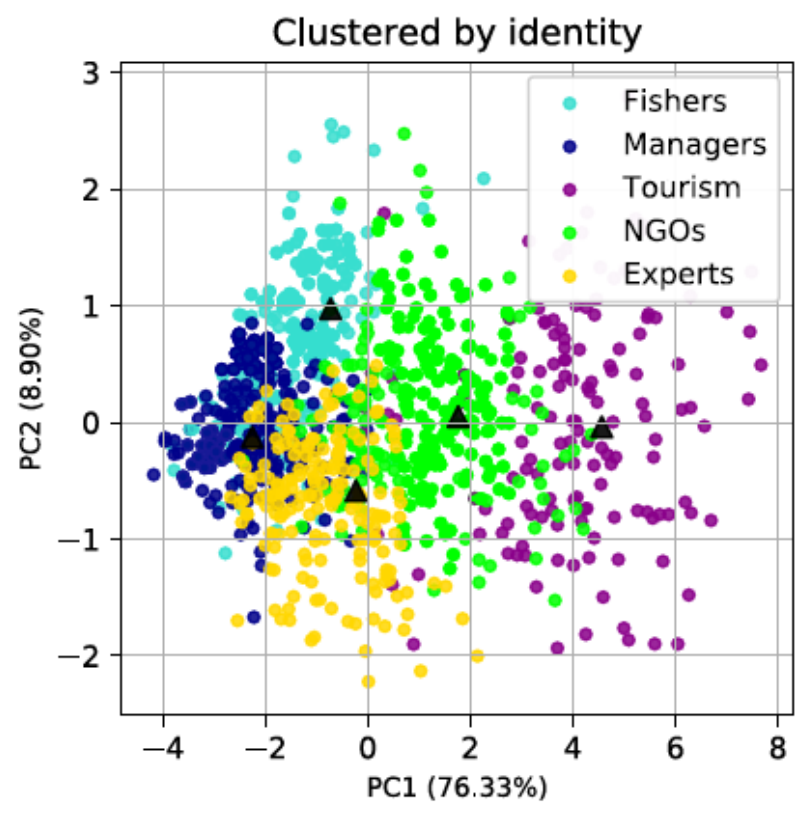

B

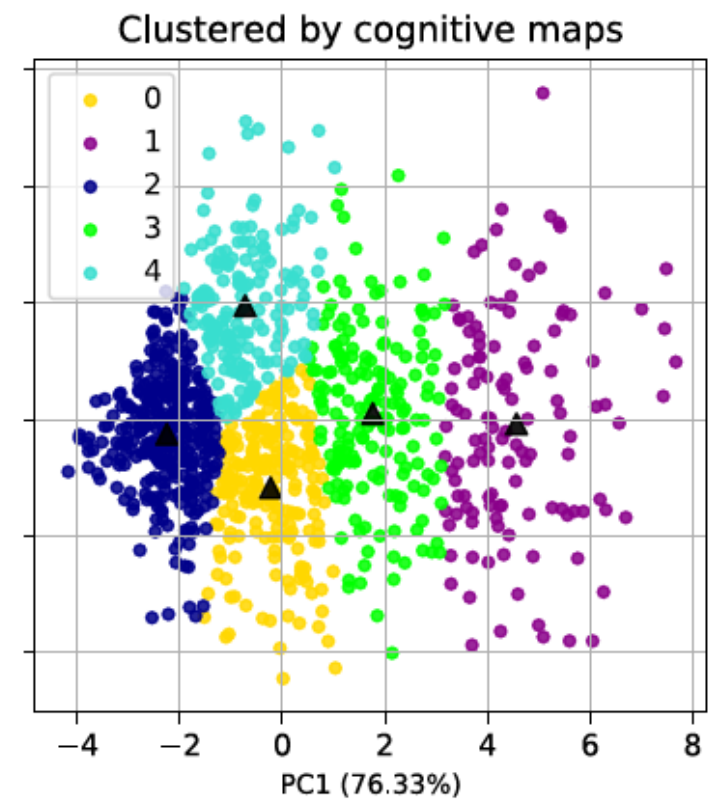

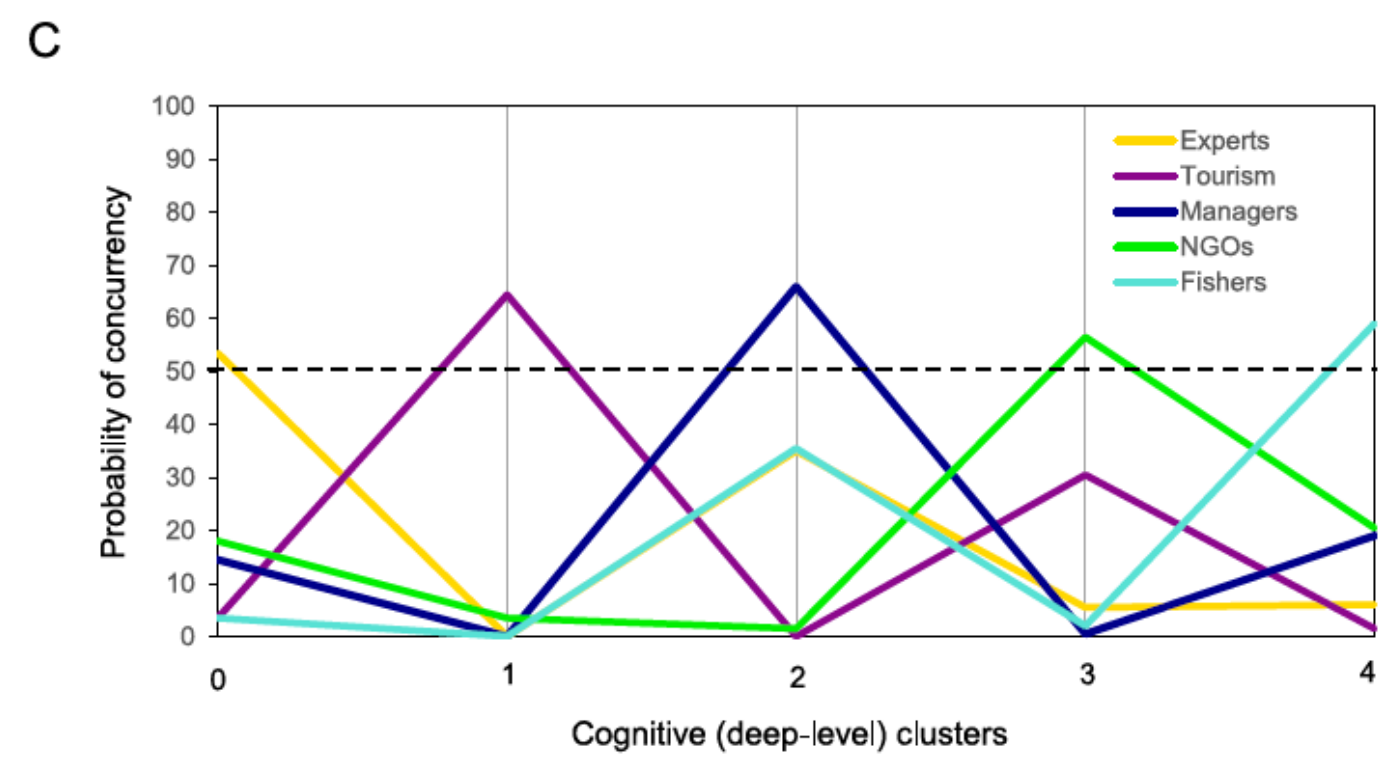

Fig 4. The proximity of identity and cognitive diversities based on micro-motifs in cognitive maps. Principle component analysis was performed on the seven dimensions of micro-motif frequencies in 1000 random cognitive maps (with 200 individual maps were reproduced form the probability distribution of cognitive maps of each of the five social groups). Two principle components were retained, cumulatively explaining about $85 \%$ of variance. Individual maps are illustrated by points in a 2-dimensional principle component scatter plot where points are clustered by their predefined social identities in (A) and by a K-Mean clustering algorithm using the Euclidian distances between points in (B). Black triangles in (A) and (B) illustrate the center of the clusters based on K-nearest neighborhood. The probability of concurrencies of social identities and cognitive clusters is shown in (C). 
Combining approaches from complex network science, graph theory, and cognitive mapping, we explored the relationship between social identity and cognitive diversity in environmental stakeholders who interact with a common pool resource system. For this, we collected stakeholders' cognitive maps using FCM—a weighted, directed graph that visualizes people's mental models that represents how each individual perceives causal interdependencies to explain the complex real world they interact with. In this study we used a case of Western Baltic cod in Germany and collected FCMs from five groups of stakeholders whose identities are socially distinguishable (i.e., social categories that are identified by their distinct roles and types of resource use which specify how they interact with the fisheries ecosystem and its resources).

Our data indicate that individuals whose identities (i.e., social categories) vary and demonstrate variations at surface-level, also develop cognitive maps that are more likely to demonstrate diverging network structural aspects as determined by their longer cognitive distances-a quantitative measure to represent cognitive, deep-level variations base on cognitive map characteristics. We developed a novel measure of cognitive distance which simultaneously takes into account dissimilarities in graph and spectral-graph metrics to provide useful insights about how individuals' cognitive maps (i.e., mental models) differ at the macro scale (metrics that represent a graph as a whole). Yet, it is also interesting to consider the variations of cognitive maps at the micro scale. To that end, we examined the distribution of directed graphlets in cognitive maps (i.e. micro-motifs) that represent common patterns of perceived causalities and are building blocks of causal reasoning (52). This micro-motif comparisons, too, showed the proximity of identity and cognitive diversities (Fig. 4).

\section{Trade-offs in measuring knowledge diversity}

Currently, there are several methods that exist to elicit and compare knowledge diversity, each with trade-offs. For example, "Cultural Consensus" theory (64) is a relatively straightforward way 
to understanding within and across group differences, often measured through evaluating individuals' responses to a series of related questions where norms and shared beliefs can be assessed through aggregate responses (65). While these methods have been widely used, many questions posed to individuals exist at a broad-level and often force participants to select binary responses (true/false). Additionally, qualitative approaches, such as applying emergent coding rubrics to concept maps or narratives are also common (66). While these approaches provide rich data, analyzing and coding qualitative concept maps take considerable time and are resources that might not be available with larger datasets. Finally, FCM as a semiquantitative assessment, such as the approach we use here, has been popular in recent years. Gray et al. (2014) point out, however, that there are considerable trade-offs in how these cognitive maps are collected: are concepts/elements in the model pre-defined?, are these maps the result of an interviewer leading the process of map development or are crowdsourced freely or are they a mix of data collection methods? Each decision a researcher makes in the data collection process will influence the analytical options available to the researchers and should be considered fully in the design of studies seeking to elicit, capture and integrate or compare individual knowledge (42).

In the context of social-ecological systems, and in contrast to our findings, Stier et al. (2017) found that experts can exhibit cognitively diverse views and perceptions about the structure of a complex ecosystem (e.g., marine food web), independent of commonly identified "bins" of expertise (e.g., local, scientific, traditional) (17). That is, the identity and cognitive diversities may not necessarily co-occur. The authors of that study have contended that individuals' demographics and background may not explain differences in perceptions of complex ecosystem structure as evidenced by lack of variations in their cognitive maps.

We argue that such findings might be influenced by the methodological biases resulting from highly standardized elicitation methods where cognitive maps are constructed using pre-defined 
standardized concepts provided by researchers. In such cases, representation of individuals' cognitive maps is significantly influenced by researchers' presumptions or limitations; consequently, true cognitive diversity is less likely to be fully captured. Therefore, we decided to provide more flexibility and elicited cognitive maps while individuals were able to freely brainstorm, represent concepts, and draw connections between them with no influence from researchers and facilitators. This decentralized process allows individuals to freely represent their internal perceptions and system knowledge, and therefore it increases the probability that a wider spectrum of knowledge diversity (i.e., cognitive coverage) is sampled. In addition, conventional methods to compare FCMs (e.g. methods described in (67)) which were used by prior studies (e.g., (17)), take into account fewer structural metrics mainly obtained by comparing the value of network global statistics, such as the density, number of receiver/driver/ordinary nodes, complexity index, hierarchy index, and the centrality of particular nodes. Except for the centrality, these metrics do not consider the correspondence between nodes - that is, two FCMs with different set of nodes, (i.e., different qualitative compositions) may be considered very similar only because they have the same number of nodes or how these nodes are connected to each other matches across two FCMs (i.e., apples and oranges considered similar because they both have round shapes). These limitations may impact the results of previous studies. Here we addressed these limitations by introducing a novel approach to measuring cognitive distances within and between groups of stakeholders.

\section{Conclusion}

In sum, our approach produces a more inclusive set of insights into understanding and measuring within group and between group knowledge variations, which has three important implications: First, measuring within group cognitive distances has implications for how we understand similarities and knowledge homogeneity within "social groups", which enables 
innovative approaches to measuring culture (shared ideas and knowledge) and group-specific cognitive biases or alternatively, identifying different types of expertise (e.g. commercial fishermen may have more expertise about biological or market-related aspects of a fishery compared to other groups). As our study supports, individuals form the same social group who interact similarly with natural resources and the ecosystems hold unique knowledge (i.e., culture), as anticipated given their shared experiences and routine class of human-environment interactions, likely shared beliefs and values, and more frequent exposure to the same information sources and social network (e.g., shared media and news outlets), thereby building in their minds cognitively more homogenous understanding of the complex ecosystem compared to the members of other social groups. Our novel approach of measuring within group shared knowledge helps us to understand how different social groups construct their specific cultural spaces about the environment which, in turn, lead stakeholders to behave in a certain way in response to environmental and social changes.

Second, measuring across group cognitive distances has implications for understanding how incorporating diverse knowledge and perceptions from across groups may ensure problemadequate solutions, reaching knowledge saturation points, and the achievement of more complete "cognitive coverage". Knowledge held by stakeholders varies across social groups, yet suggesting that different types of stakeholders hold complementary perceptions of complex social-ecological interdependencies. Our approach of measuring between group cognitive distances ensures that we bring in adequate knowledge diversity from across multiple groups to harness their collective intelligence. While we did not evaluate whether more diverse groups improve group task performance (13), our data does indicate that surface and deep diversity are correlated with improved measures.

Finally, our findings have applications for designing inclusive processes and adaptive comanagement practices (23). Such approaches encourage the participation and involvement of 
relevant stakeholders and may enhance the credibility and legitimization of management strategies while resource users, managers, NGOs, policymakers, and scientists bridge their divides and jointly agree on possible management actions for uncertain ecosystems (68). Furthermore, to achieve knowledge co-production, inclusive processes with buy-in from diverse individuals should also guarantee an increase in the total pool of available knowledge and cognitive coverage. Our study assures that involving diverse groups of stakeholders into adaptive co-management can also achieve knowledge co-production: the "Iterative and collaborative processes involving diverse types of expertise, knowledge and actors to produce context-specific knowledge and pathways towards a sustainable future" (24).

\section{Acknowledgments}

The authors like to sincerely thank all stakeholders for their participation in this study, only through tehir cooperation the project could be carried out in this way. This research was (in part) supported by PhD Scholarship Programme of the German Federal Environmental Foundation (Deutsche Bundesstiftung Umwelt, Osnabrueck; no. 2017/480) to HS. 


\section{References}

1. Page SE. Making the difference: Applying a logic of diversity. Acad Manag Perspect. 2007;21(4):6-20.

2. Phillips KW, Loyd DL. When surface and deep-level diversity collide: The effects on dissenting group members. Organ Behav Hum Decis Process. 2006;99(2):143-60.

3. de Oliveira S, Nisbett RE. Demographically diverse crowds are typically not much wiser than homogeneous crowds. Proc Natl Acad Sci. 2018;115(9):2066-71.

4. Page SE. The diversity bonus: How great teams pay off in the knowledge economy. Princeton University Press; 2019.

5. Page SE. The difference: How the power of diversity creates better groups, firms, schools, and societies-new edition. Princeton University Press; 2008.

6. Hong L, Page SE. Groups of diverse problem solvers can outperform groups of highability problem solvers. Proc Natl Acad Sci. 2004;101(46):16385-9.

7. Baggio JA, Freeman J, Coyle TR, Nguyen TT, Hancock D, Elpers KE, et al. The importance of cognitive diversity for sustaining the commons. Nat Commun. 2019;10(1):1-11.

8. Aminpour P, Gray S, Singer A, Scyphers S, Jetter A, Jordan R, et al. The diversity bonus in pooling local knowledge about complex problems. Proc Natl Acad Sci U S A. 2021; 118(5) e2016887118.

9. Kao AB, Berdahl AM, Hartnett AT, Lutz MJ, Bak-Coleman JB, loannou CC, et al. Counteracting estimation bias and social influence to improve the wisdom of crowds. J R Soc Interface. 2018;15(141):20180130.

10. Bernstein E, Shore J, Lazer D. How intermittent breaks in interaction improve collective intelligence. Proc Natl Acad Sci. 2018;115(35):8734-9.

11. Reynolds A, Lewis D. Teams solve problems faster when they're more cognitively diverse. Harv Bus Rev. 2017;30.

12. Horwitz SK, Horwitz IB. The effects of team diversity on team outcomes: A meta-analytic review of team demography. J Manage. 2007;33(6):987-1015.

13. Woolley AW, Chabris CF, Pentland A, Hashmi N, Malone TW. Evidence for a collective intelligence factor in the performance of human groups. Science (80- ). 2010;330(6004):686-8.

14. Camara WJ, Nathan JS, Puente AE. Psychological test usage: Implications in professional psychology. Prof Psychol Res Pract. 2000;31(2):141.

15. Johnson-Laird PN. Mental models and human reasoning. Proc Natl Acad Sci. 2010;107(43):18243-50.

16. Chan D, Schmitt N, DeShon RP, Clause CS, Delbridge K. Reactions to cognitive ability tests: the relationships between race, test performance, face validity perceptions, and test-taking motivation. J Appl Psychol. 1997;82(2):300. 
17. Stier AC, Samhouri JF, Gray S, Martone RG, Mach ME, Halpern BS, et al. Integrating expert perceptions into food web conservation and management. Conserv Lett. 2017;10(1):67-76.

18. Stern PC, Dietz T. Public participation in environmental assessment and decision making. National Academies Press Washington, DC; 2008.

19. Jackson SE, Ruderman MN. Diversity in work teams: Research paradigms for a changing workplace. American Psychological Association; 1995.

20. Gaither SE, Apfelbaum EP, Birnbaum HJ, Babbitt LG, Sommers SR. Mere membership in racially diverse groups reduces conformity. Soc Psychol Personal Sci. 2018;9(4):402-10.

21. Kearney E, Gebert D, Voelpel SC. When and how diversity benefits teams: The importance of team members' need for cognition. Acad Manag J. 2009;52(3):581-98.

22. Adams WM, Brockington D, Dyson J, Vira B. Managing tragedies: understanding conflict over common pool resources. Science (80- ). 2003;302(5652):1915-6.

23. Armitage D, Berkes F, Doubleday N. Adaptive co-management: collaboration, learning, and multi-level governance. UBC Press; 2010.

24. Norström A V, Cvitanovic C, Löf MF, West S, Wyborn C, Balvanera P, et al. Principles for knowledge co-production in sustainability research. Nat Sustain. 2020;1-9.

25. Aminpour P, Gray SA, Jetter AJ, Introne JE, Singer A, Arlinghaus R. Wisdom of stakeholder crowds in complex social--ecological systems. Nat Sustain. 2020;1-9.

26. Levin S, Xepapadeas T, Crépin AS, Norberg J, De Zeeuw A, Folke C, et al. Socialecological systems as complex adaptive systems: Modeling and policy implications. Environ Dev Econ. 2013 Apr;18(2):111-32.

27. Ostrom E. A general framework for analyzing sustainability of social-ecological systems. Science (80- ). 2009;325(5939):419-22.

28. Freeman J, Baggio JA, Coyle TR. Social and general intelligence improves collective action in a common pool resource system. Proc Natl Acad Sci. 2020;117(14):7712-8.

29. Gray S, Aminpour P, Reza C, Scyphers S, Grabowski J, Murphy JR, et al. Harnessing the Collective Intelligence of Stakeholders for Conservation. Front Ecol Environ. 2020;

30. St John FA V, Steadman J, Austen G, Redpath SM. Value diversity and conservation conflict: Lessons from the management of red grouse and hen harriers in England. People Nat. 2019;1(1):6-17.

31. Otto-Banaszak I, Matczak P, Wesseler J, Wechsung F. Different perceptions of adaptation to climate change: a mental model approach applied to the evidence from expert interviews. Reg Environ Chang. 2011;11(2):217-28.

32. Voss R, Quaas MF, Stiasny MH, Hänsel M, Pinto GASJ, Lehmann A, et al. Ecologicaleconomic sustainability of the Baltic cod fisheries under ocean warming and acidification. J Environ Manage. 2019;238:110-8.

33. Funk S, Krumme U, Temming A, Möllmann C. Gillnet fishers' knowledge reveals seasonality in depth and habitat use of cod (Gadus morhua) in the Western Baltic Sea. ICES J Mar Sci. 2020; 
34. McQueen K, Casini M, Dolk B, Haase S, Hemmer-Hansen J, Hilvarsson A, et al. Regional and stock-specific differences in contemporary growth of Baltic cod revealed through tag-recapture data. ICES J Mar Sci. 2020;

35. Kosko B. Fuzzy cognitive maps. Int J Man Mach Stud. 1986;24(1):65-75.

36. Jetter AJ, Kok K. Fuzzy Cognitive Maps for futures studies-A methodological assessment of concepts and methods. Futures. 2014;61:45-57.

37. Mohammed S, Dumville BC. Team mental models in a team knowledge framework: Expanding theory and measurement across disciplinary boundaries. J Organ Behav Int $\mathrm{J}$ Ind Occup Organ Psychol Behav. 2001;22(2):89-106.

38. Johnson-Laird PN. Mental models: Towards a cognitive science of language, inference, and consciousness. Harvard University Press; 1983.

39. Eden C. On the nature of cognitive maps. J Manag Stud. 1992;29(3):261-5.

40. Gray SA, Gray S, De Kok JL, Helfgott AER, O'Dwyer B, Jordan R, et al. Using fuzzy cognitive mapping as a participatory approach to analyze change, preferred states, and perceived resilience of social-ecological systems. Ecol Soc. 2015;20(2).

41. Savić M, Ivanović M, Jain LC. Fundamentals of Complex Network Analysis. In: Complex Networks in Software, Knowledge, and Social Systems [Internet]. Cham: Springer International Publishing; 2019. p. 17-56. Available from: https://doi.org/10.1007/978-3319-91196-0_2

42. Gray SA, Zanre E, Gray SRJ. Fuzzy cognitive maps as representations of mental models and group beliefs. In: Fuzzy cognitive maps for applied sciences and engineering. Springer; 2014. p. 29-48.

43. Schwermer H, Aminpour P, Reza C, Funk S, Möllmann C, Gray SA. Modeling and Understanding Social-Ecological Knowledge Diversity. Conserv Sci Pract.

44. Tantardini M, leva F, Tajoli L, Piccardi C. Comparing methods for comparing networks. Sci Rep. 2019;9(1):1-19.

45. Cohen-Steiner D, Kong W, Sohler C, Valiant G. Approximating the spectrum of a graph. In: Proceedings of the 24th acm sigkdd international conference on knowledge discovery \& data mining. 2018. p. 1263-71.

46. Chung FRK, Graham FC. Spectral graph theory. American Mathematical Soc.; 1997.

47. Bollobás B. Modern graph theory. Vol. 184. Springer Science \& Business Media; 2013.

48. Newman M. Networks: An Introduction [Internet]. OUP Oxford; 2010. Available from: https://books.google.com/books?id=-DgTDAAAQBAJ

49. Koutra D, Parikh A, Ramdas A, Xiang J. Algorithms for graph similarity and subgraph matching. In: Proc Ecol Inference Conf. 2011.

50. Gera R, Alonso L, Crawford B, House J, Mendez-Bermudez JA, Knuth T, et al. Identifying network structure similarity using spectral graph theory. Appl Netw Sci. 2018;3(1):2.

51. Shannon CE. A mathematical theory of communication. Bell Syst Tech J. 1948;27(3):379-423. 
52. Levy MA, Lubell MN, McRoberts $N$. The structure of mental models of sustainable agriculture. Nat Sustain. 2018;1(8):413-20.

53. Grotzer TA, Shane Tutwiler M. Simplifying Causal Complexity: How Interactions Between Modes of Causal Induction and Information Availability Lead to Heuristic-Driven Reasoning. Mind, Brain, Educ [Internet]. 2014 Sep 1 [cited 2020 May 2];8(3):97-114. Available from: http://doi.wiley.com/10.1111/mbe.12054

54. White PA. Naive ecology: Causal judgments about a simple ecosystem. Br J Psychol [Internet]. 1997 May 1 [cited 2020 May 2];88(2):219-33. Available from: http://doi.wiley.com/10.1111/j.2044-8295.1997.tb02631.x

55. Trope Y, Liberman N. Construal-Level Theory of Psychological Distance. Psychol Rev. 2010 Apr;117(2):440-63.

56. Halbrendt J, Gray SA, Crow S, Radovich T, Kimura AH, Tamang BB. Differences in farmer and expert beliefs and the perceived impacts of conservation agriculture. Glob Environ Chang. 2014;28:50-62.

57. Olazabal M, Chiabai A, Foudi S, Neumann MB. Emergence of new knowledge for climate change adaptation. Environ Sci Policy. 2018;83:46-53.

58. Hamilton M, Salerno J, Fischer AP. Cognition of complexity and trade-offs in a wildfireprone social-ecological system. Environ Res Lett. 2019;14(12):125017.

59. Oliver TH, Heard MS, Isaac NJB, Roy DB, Procter D, Eigenbrod F, et al. Biodiversity and resilience of ecosystem functions. Trends Ecol Evol. 2015;30(11):673-84.

60. Duchek S, Raetze S, Scheuch I. The role of diversity in organizational resilience: a theoretical framework. Bus Res. 2020;13(2):387-423.

61. O'Brien KR, Scheffer M, Van Nes EH, der Lee R. How to break the cycle of low workforce diversity: A model for change. PLoS One. 2015;10(7):e0133208.

62. Potvin DA, Burdfield-Steel E, Potvin JM, Heap SM. Diversity begets diversity: A global perspective on gender equality in scientific society leadership. PLoS One. 2018;13(5):e0197280.

63. Gray S, Chan A, Clark D, Jordan R. Modeling the integration of stakeholder knowledge in social--ecological decision-making: benefits and limitations to knowledge diversity. Ecol Modell. 2012;229:88-96.

64. Romney AK, Weller SC, Batchelder WH. Culture as consensus: A theory of culture and informant accuracy. Am Anthropol. 1986;88(2):313-38.

65. Paolisso M. Cultural models and cultural consensus of Chesapeake Bay blue crab and oyster fisheries. Napa Bull. 2007;28(1):123-35.

66. Greene BA, Lubin IA, Slater JL, Walden SE. Mapping changes in science teachers' content knowledge: Concept maps and authentic professional development. J Sci Educ Technol. 2013;22(3):287-99.

67. Özesmi U, Özesmi SL. Ecological models based on people's knowledge: a multi-step fuzzy cognitive mapping approach. Ecol Modell. 2004;176(1-2):43-64.

68. Plummer R, Crona B, Armitage DR, Olsson P, Tengö M, Yudina O. Adaptive comanagement: a systematic review and analysis. Ecol Soc. 2012;17(3). 